not the neonatal scan, we speculate that NS may have a negative effect on hippocampal development. However, these results could also be explained by unmeasured neoantal hypoxic-ischemic injury. The findings are in keeping with studies that suggest NS are an independent risk factor for adverse neurodevelopmental outcome. Further studies are needed to confirm whether seizures harm newborns.

Acknowledgements NIH/NCRR (UL1 RR024131); NIH/NINDS P50NS035902

\section{INVESTIGATE OF S100B PROTEIN IN SERUM AS PROGNOSTIC MARKER FOR BRAIN INJURY IN TERM NEWBORN INFANTS WITH HYPOXIC ISCHEMIC ENCEPHALOPATHY}

doi:10.1136/archdischild-2012-302724.1087

${ }^{1}$ AN Sofijanova, ${ }^{2 K}$ Piperkova, ${ }^{1}$ OV Jordanova. 'Neonatal and Pediatric Intensive Care; 2Department of Neonatology, University Children's Hospital, Skopje, FYR Macedonia

Background Further to investigate whether increased S100 levels in serum are correlated with the grade of HIE after perinatal asphyxia, mechanical ventilation in some severe cases of the asphyxiated infants and more specifically whether increased S100 predicts the cerebral injury and subsequent cerebral palsy.

Methods All risk neonates with severe asphyxia, within $24 \mathrm{~h}$ of injury were included. Serum S100 was measured on postnatal days 1-3-7 in 62 term infants with birth asphyxia. S100B levels were measured using ECLIA method.

Results The avarage serum S100B levels for the control group(N=48) was 0.12 microgL(-1) (cut-off point). S100B levels were significantly higher in asphyxiated term neonates $N=29 ; M=$ 0.64. Infants with moderate and severe HIE had significantly higher S100 levels on postnatal day $1(p=0.031)$ and day $2(p=0.008)$ than infants with mild or no HIE. The levels of S100 decreased on days 2 and 3 in all infants with HIE. The median S100 level on postnatal day 1 was higher in nine infants who died neonatally and in 10 infants who developed cerebral palsy (CP), compared with 43 infants with no signs of impairment at follow up, $14.0 \mu \mathrm{g} / \mathrm{L}$, $20.7 \mu \mathrm{g} / \mathrm{L}$ and $5.5 \mu \mathrm{g} / \mathrm{L}$, respectively. A level of S100 above $12 \mu \mathrm{g} / \mathrm{L}$ the first day of life was significantly more frequent in infants who died or developed CP than in infants with no impairment at follow up $(p=0.02)$.

Conclusion Early determination of serum S100 may reflect the extent of brain damage in infants with HIE after asphyxia.

\section{VENTILATION MODALITY AND CEREBRAL DAMAGE IN PRETERM INFANTS}

doi:10.1136/archdischild-2012-302724.1088

A Arco, GT Pagano, E Gitto, S Aversa, I Barberi. Pediatric, University of Messina, Messina, Italy

Background and Aims Several studies on high frequency oscillatory ventilation (HFOV) have demonstrated a high relation between this method and the incidence of hemorrhagic or ischemic brain injures. In this study, we meant to verify the incidence of intraperiventricular hemorrhage (III-IV stadium) and cystic periventricular leukomalacia (CPVL) in subjects submitted to different ventilation (HFOV vs CMV).

Materials and Methods We have examined 120 newborns (mean gestational age $30 \pm 1.85$ weeks, mean birth weight $1314 \pm 404.14$ grams). Group A: 60 infants ventilated in HFOV; Group B: 60 infants ventilated in $\mathrm{CMV}$. All received surfactant.

All infants underwent monitoring of cardiovascular function with evaluation of parameters such as fractional shortening, ejection fraction, size of rooms, the ductal shunt, pulmonary artery pressure and cardiac output and blood pressure.
Results Infants ventilated in HFOV have required a maximum value of MAP significantly lower $(p<0.05)$ versus those ventilated in CMV; Iin group A, 8 (13.3\%) experienced a hemorrhage of III-IV degree against $12(20 \%)$ of subjects in group $\mathrm{B}$.

They presented CPVL $14(7.23 \%)$ infants in group A compared with $16(26.6 \%)$ in group B. There were no differences in cerebral blood flow and resistance index of the anterior cerebral artery. There were no differences in cardiac function.

Conclusions Our data show an increase, not statistically significant, of PIVH and CPVL in newborn infants treated with conventional ventilation than oscillatory ventilation. This is attributable to the use of a MAP "optimal" able to obtain a good alveolar recruitment without causing hyperexpansion.

\section{VISUAL EVOKED POTENTIALS IN TERM AND PRETERM INFANTS}

doi:10.1136/archdischild-2012-302724.1089

F Brackmann, S Scheu, R Trollmann. Department of Pediatrics, University of ErlangenNürnberg, Erlangen, Germany

Background and Aims Evoked potentials are a useful noninvasive method for the assessment of neurological status in term and preterm infants at risk for perinatal CNS damage. The present study intended to gather reference values of visual evoked potentials (VEP) for preterm and term neonates and identify neonates at risk for perinatal CNS lesions using VEPs.

Methods 23 healthy preterm and term neonates (group A) and 16 infants with perinatal brain injury (group B) were examined for this study. Groups were classified according to their post-conceptual age (A1/B1: 42-45 weeks, n=4/6; A2/B2: 38-41 weeks, n=5/8; A3: 36-37 weeks, $n=12 ; A 4 / B 3:<36$ weeks, $n=2 / 2)$. Stimulation was triggered by stroboscopic flashes $(1 \mathrm{~Hz} / 10 \mu \mathrm{s})$ and $\mathrm{P} 1$ and $\mathrm{N} 2$ waves were analyzed.

Results Latencies significantly correlated with post-conceptual age ( $\mathrm{P} 1: \mathrm{p}<0.001, \mathrm{~N} 2: \mathrm{p}<0.05)$ and gestational age $(\mathrm{P} 1: \mathrm{p}<0.01)$. The average latency values (mean $\pm \mathrm{SD}$ ) of the subgroups were: group A1 (P1:165.7ms \pm 33.5 ; N2:211.5ms \pm 29.9 ), A2 (P1: 199.6ms \pm 34.2 ; $\mathrm{N} 2: 255.6 \mathrm{~ms} \pm 25.8)$, A3 (P1:223.8ms $\pm 14.7 ; \mathrm{N} 2: 272.1 \mathrm{~ms} \pm 13.5)$, A4 (P1:240 resp. 209ms; N2:242 resp. 233ms). Average latencies of term infants with or without perinatal injury differed significantly for P1 (228.8 ms \pm 30.9 vs. $165.7 \mathrm{~ms} \pm 33.5 ; \mathrm{p}<0.05)$ and $\mathrm{N} 2(266.0$ $\mathrm{ms} \pm 21.1$ vs. $211.5 \mathrm{~ms} \pm 29.9 ; \mathrm{p}<0.01$ ).

Conclusions The present study adds to the knowledge on normal VEP values during early development. Present data showed a negative correlation with post-conceptual age for central latencies as an equivalent of progressing myelination regardless of extra- or intrauterine maturation. Term infants with perinatal brain injury showed significantly prolonged VEP latencies compared to healthy children.

\section{RELATIONSHIP BETWEEN NEUROTROPHINS AND BRAIN STRUCTURE IN PRETERM GROWTH RESTRICTED BABIES}

doi:10.1136/archdischild-2012-302724.1090

'L Parkes, ${ }^{2} \mathrm{~J}$ Atkinson, ${ }^{3} \mathrm{~J}$ Miyan, ${ }^{4} \mathrm{~A}$ Hendrickson, $4,5 \mathrm{~S}$ Victor. ${ }^{1}$ Cancer and Enabling Sciences, University of Manchester, Manchester, ${ }^{2}$ School of Health Sciences, University of Liverpool, Liverpool; ${ }^{3}$ Faculty of Life Sciences, University of Manchester; ${ }^{4}$ Newborn Intensive Care Unit, Central Manchester University Hospitals NHS Foundation Trust; ${ }^{5}$ School of Biomedicine, University of Manchester, Manchester, UK

Background Neurotrophins are responsible for the growth and survival of neurons during early brain development. Intrauterine growth restriction (IUGR) leads to alterations in brain structure. Aim To explore the relationship between neurotrophins and brain structure in preterm IUGR babies. 
Methods 31 babies born between 28 and 36 weeks' gestation were studied. 19 babies were IUGR with individualised birth weight (normalised for sex, ethnicity, parity, maternal BMI) below $3^{\text {rd }}$ centile. 12 babies were appropriately grown with individualised birth weight between the $25^{\text {th }}$ and $75^{\text {th }}$ centile. Blood neurotrophin concentrations were measured using protein chip technology in 30 babies (19 IUGR and 11 controls) between 2 and 7 days after birth. In 14 babies ( 7 from each group) MRI brain was performed at term equivalence.

Results Fractional anisotropy (FA) was lower in IUGR babies compared to controls in 7 out of 8 regions with no statistical significance. Apparent diffusion coefficient (ADC) was lower in IUGR babies compared to controls in 6 out of 8 regions, reaching significance in frontal lobes. 7 out of 8 regions were smaller in IUGR babies compared to the control babies, reaching significance in the dorso-medial pre-frontal cortex. Differences did not persist when normalised for intracranial volume. Serum neurotrophin concentrations were elevated in IUGR babies but did not reach statistical significance. Using multiple regression only FA of right frontal lobe was significantly related to $B D N F(R=0.65$; $\mathrm{p}=0.012$ )

Conclusion IUGR babies showed no differences in neurotrophin concentrations and decreased $\mathrm{ADC}$ in frontal lobes when compared with controls.

\section{PREDICTIVE VALUE OF VASCULAR ENDOTHELIAL GROWTH FACTOR IN PRETERM NEONATES WITH INTRAVENTRICULAR HAEMORRHAGE}

doi:10.1136/archdischild-2012-302724.1091

S Atef, S Hassanin, M El Chimi, M Mohamed. Ain Shams University, Cairo, Egypt

Objective Intraventricular haemorrhage (IVH) is a major problem in premature infants. Our objective is to assess the early predictive value of vascular endothelial growth factor (VEGF) for development of IVH and management of its squeal in preterm neonates.

Methods We prospectively studied 150 preterm neonates (PT) less than 34 weeks gestation. Fifty of them completed the study. 30/50 developed IVH during follow up, and 20 did not. First 24 hours, and 3rd day serum samples were collected. Cerebrospinal fluid (CSF) samples were withdrawn for $10 \mathrm{IVH}$ patients.

Results Serum VEGF; both samples were increased in IVH compared to non-IVH group, $(\mathrm{P}=0.001)$. PHVD-group $(\mathrm{n}=10)$ had higher VEGF in both samples than resolved IVH $(\mathrm{P}=0.004),(\mathrm{P}=0.005)$. While, VEGF increased in the IVH group 2nd sample compared to 1 st $(\mathrm{P}=0.000)$, it decreased in non-IVH group, $\mathrm{P}=0.033)$. Each 1 unit increase in 1ST VEGF increased the risk of occurrence of IVH by $1.6 \%$. 3rd day VEGF at a cut-off value of $135 \mathrm{pg} / \mathrm{ml}$ is $96 \%$ sensitive and 100 specific to predict post haemorrhagic ventricular dilatation (PHVD). Serum VEGF inversely correlated with TLC, pH, PO2 and $\mathrm{HCO} 3$, and positively correlated with $\mathrm{PCo} 2$ and $\mathrm{FiO} 2$.

Conclusion Serum VEGF predicts development of IVH and PHVD in PT neonates. Also, high CSF level of VEGF could predict the need for permanent shunt placement.

\section{NEONATAL HYPOGLYCEMIA IS ASSOCIATED WITH INCREASED ALBUMIN NITRATION IN SMALL FOR GESTATIONAL AGE TERM NEWBORNS}

doi:10.1136/archdischild-2012-302724.1092

'N Lefevre, ' $\mathrm{JL}$ Wayenberg, ${ }^{2} \mathrm{C}$ Ghaddhab, ${ }^{2} \mathrm{D}$ Vermeylen, ${ }^{3} \mathrm{E}$ Damis, ${ }^{3} \mathrm{M}$ Flausch, ${ }^{4} \mathrm{~S}$ Bottari. 'Department of Pediatrics, Hôpital Universitaire des Enfants Reine Fabiola; ${ }^{2}$ Neonatal Intensive and Non-Intensive Care Unit, Hôpital Erasme; ${ }^{3}$ Neonatal Intensive and Non-Intensive Care Unit, CHIREC, Brussels, Belgium; ${ }^{4}$ Department of Biology and Pathology, CHU \& Laboratoire de Bioénergétique Fondamentale et Appliquée - INSERM U1055, Université Joseph Fourier, Grenoble, France
Background and Aims Neonatal hypoglycemia is a frequent event in small for gestational age (SGA) term newborns. Its clinical significance is a highly controversial issue but in experimental models, hypoglycemia has been reported to cause oxidative stress. Among the reactive species, peroxynitrite is responsible for protein nitration, lipid peroxydation and DNA damage, a process referred to as nitro-oxidative stress which can induce apoptosis. The aim of the present study was to investigate whether hypoglycemia is associated with plasma albumin nitration as a marker of nitro-oxidative stress in SGA term newborns.

Methods Using a highly sensitive ELISA we quantified plasma nitroalbumin (PNA) as a marker of peroxynitrite generation in 26 SGA term newborns with close glucose monitoring. We compared PNA concentrations in 9 normoglycemic (glycemia $\geq 2.5 \mathrm{mmol} / \mathrm{L}$ ) newborns and in 17 hypoglycemic (glycemia $<2.5 \mathrm{mmol} / \mathrm{L}$ ) newborns.

Results PNA measured during the first hours of life was inversely correlated with glycemia $(r=-0.63, p=0.01)$ and significantly higher in hypoglycemic compared to normoglycemic patients $(7.4 \mathrm{ng} / \mathrm{mL}$ [5.0-7.9] in normoglycemic patients vs. $20.8 \mathrm{ng} / \mathrm{mL}$ [9.9-77.7] in hypoglycemic patients, $\mathrm{n}=14, \mathrm{p}<0.01)$. PNA measured at day 1 of life was significantly higher in patients with recurrent hypoglycemia compared to patients with transient hypoglycemia and normoglycemic patients. We observed significant correlations between PNA at day 1 and the area under the curve of the glycemia measured before PNA sampling for several threshold values of glycemia.

Conclusions These results indicate that recurrent hypoglycemia is associated with systemic protein nitration in SGA term newborns, suggestive of a significant nitro-oxidative stress.

\section{HYPOGLYCEMIA INDUCES A NITRO-OXIDATIVE STRESS IN} PRETERM NEWBORNS

doi:10.1136/archdischild-2012-302724.1093

${ }^{1} \mathrm{C}$ Ghaddhab, ${ }^{1} \mathrm{~N}$ Lefèvre, ${ }^{1} \mathrm{JL}$ Wayenberg, ${ }^{2,3} \mathrm{SP}$ Bottari. 'Department of Pediatrics, Université Libre de Bruxelles, Brussels, Belgium; ${ }^{2}$ Fundamental and Applied Bioenergetics (LBFA), INSERM U1055, and Université Joseph Fourrier, Grenoble, St Martin d'Heres; ${ }^{3}$ Medicinal Chemistry, CHU Grenoble, Grenoble, France

Recent data suggest that free radical injury occurs in the neonatal brain after hypoglycemia in animal models. We developed an ELISA allowing the quantitative determination of nitrated plasma albumin (nitroalbumin, PNA) as a biomarker of peroxynitrite generation and investigated the potential nitro-oxidative stress induced by hypoglycemia $(<2.5 \mathrm{mmol} / \mathrm{L})$ in premature newborns.

We measured PNA concentrations at days 0,1 and 4 of life in 72 preterm infants without any other obvious cause of nitro-oxidative stress such as infection, asphyxia and hyperglycemia. Glucose levels were monitored every 3-4 hours using a strip method. For each patient, we calculated the AUCs for glycaemia measured during the 12,18 and 24 hours preceding blood sampling as an index of the severity, the number and the duration of hypoglycemic events during the first day of life. Statistical analysis was performed using non-parametric tests.

PNA concentrations were significantly higher in hypoglycaemic than in normoglycemic infants at days 0,1 and 4. A significant inverse correlation was found between PNA at $\mathrm{D}_{1}$ and AUGs. PNA concentration at $D_{1}$ is related to the number of hypoglycemic events. Gender, term, oxygen exposure, respiratory and hemodynamic parameters were not correlated with PNA concentrations.

Thus, low glycemia levels during the first day of life are specifically associated with increased albumin nitration in preterm newborns, especially in case of recurrent hypoglycemia. This suggests the occurrence of systemic nitro-oxidative stress implying a risk of end-organ damage due to protein nitration, lipid peroxidation and DNA damage, in particular to the brain. 